\title{
Noradrenaline and dobutamine effects on the volume expansion with normal saline in rabbits subjected to hemorrhage ${ }^{1}$
}

\author{
Gualter Lisboa Ramalho ${ }^{\mathrm{I}}$, Matheus Fachini Vane ${ }^{\mathrm{II}}$, Luciana Cavalcanti Lima ${ }^{\mathrm{III}}$, Lucas Fachini Vane ${ }^{\mathrm{IV}}$, Rosa Beatriz Amorim ${ }^{\mathrm{V}}$, \\ Maria Aparecida Domingues $^{\mathrm{VI}}$, José Mariano Soares de Moraes ${ }^{\mathrm{VII}}$, Lídia Raquel de Carvalho ${ }^{\mathrm{VIII}}$, Pedro Paulo Tanaka ${ }^{\mathrm{IX}}$, Luiz \\ Antonio Vane ${ }^{X}$ \\ DOI: http://dx.doi.org/10.1590/S0102-865020160090000008 \\ ${ }^{\mathrm{I}}$ Fellow PhD degree, Postgraduate Program in Anesthesiology, Botucatu Medical School, Universidade Estadual Paulista (UNESP), Botucatu-SP, \\ Brazil. Conception, design, intellectual and scientific content of the study. \\ "MD, Division of Anesthesiology, Medical School, University de São Paulo (USP), Brazil. Conception of the study, manuscript writing. \\ IIIPhD, Pernambuco Faculty of Health, Recife-PE, Brazil. Manuscript writing. \\ ${ }^{\text {IV }}$ Master, Guaratingueta School of Engineering, UNESP, Brazil. Acquisition of data. \\ ${ }^{v} \mathrm{PhD}$, Department of Anesthesiology, Botucatu Medical School, UNESP, Brazil. Technical procedures. \\ VIPhD, Department of Pathology, Botucatu Medical School, UNESP, Brazil. Histological examinations. \\ VIIPhD, Division of Anesthesiology, Faculdade de Ciências Médicas e da Saúde de Juiz de Fora, Brazil. Manuscript writing, critical revision. \\ ${ }^{V I I I} \mathrm{PhD}$, Department of Biostatistics, Institute of Biosciences, UNESP, Brazil. Statistical analysis. \\ ${ }^{\mathrm{Ix}} \mathrm{PhD}$, Department of Anesthesiology, Stanford University, Medical School, California, USA. Critical revision. \\ ${ }^{x}$ Full Professor, Department of Anesthesiology, Botucatu Medical School, UNESP, Brazil. Manuscript writing, critical revision, supervised all phases \\ of the study.
}

\section{ABSTRACT}

PURPOSE: To evaluate the effects of dobutamine (DB), noradrenaline (NA), and their combination (NADB), on volume retention in rabbits submitted to hemorrhage.

METHODS: Thirty six rabbits were randomly divided into 6 groups: SHAM, Control, Saline, DB, NA, DB+NA. All the animals, except for SHAM, were subjected to hemorrhage of $25 \%$ of the calculated blood volume. Control animals were replaced with their own blood. The other groups received NSS 3 times the volume withdrawn. The intravascular retention, hematocrit, diuresis, central venous pressure, mean arterial pressure, NGAL, dry-to-wet lung weight ratio (DTWR) and the lung and kidney histology were analyzed.

RESULTS: Replacement with NSS and NA, DB or NA+DB did not produce differences in the intravascular retention. After hemorrhage, the animals presented a significant decrease in the MAP and CVP, which were maintained until volume replacement. Regarding NGAL, dry-to-wet-lung-weight ratio, lung and kidney histology, there were no statistical differences between the groups.

CONCLUSION: The use of noradrenaline, dobutamine or their combination did not increase the intravascular retention of volume after normal saline infusion.

Key words: Plasma Volume. Hemorrhage. Catecholamines. Rabbits. 


\section{Introduction}

The infusion of crystalloid solutions is a routine treatment in patients undergoing anesthetic and surgical procedures because it is considered safe and it is of low cost ${ }^{1}$. It has a great importance in the correction of hypovolemia during bleeding, increasing blood pressure (BP), cardiac output (CO) and helping in the restoration of perfusion and tissue oxygenation ${ }^{2}$. However, the crystalloids retain their volume poorly once they are intravascular, with only $20 \%$ of the total infused volume remaining in the cardiovascular system after 20 minutes of infusion. Therefore, at least, three times the amount of blood lost should be replaced with crystaloids ${ }^{3}$. Of the remainder left in the intravascular, about half is eliminated as urine and the other half will form interstitial edema ${ }^{4}$. As a result, there is a great amount of fluid draining into the extravascular space with formation of interstitial edema. This decreases the pulmonary gas exchange, and worsens the cardiac and gastrointestinal functions, wound healing, and the cerebral blood flow ${ }^{5,6}$.

During hypovolemia due to bleeding, vasoactive drugsmany of these with inotropic and vasopressor properties-are commonly administered along with the infusion of normal saline (NSS) to improve the cardiovascular response to volume depletion $^{1}$; this allows better maintenance of cardiac output (CO), mean arterial pressure (MAP) and urine output (UO) $)^{2,7,8}$ The use of vasoactive agents along with crystalloids maintains tissue perfusion and improves cardiac contractility as well as vascular tonus, thus improving the ability to resuscitate patients in hemorrhagic shock ${ }^{9,10}$.

It is known that some catecholamines may modify the expansion of plasma volume after NSS infusion. Vane et al. ${ }^{11}$ have shown that isoproterenol - a specific $\beta$-adrenergic agonist — and dopamine - a non-specific catecholamine that is an adrenergic and dopaminergic agonist-increase vascular retention, whereas phenylephrine - a specific $\alpha$-adrenergic drug-attenuates the increase of plasma volume, and increases diuresis. On the other hand, Kinsky et al. ${ }^{12}$ demonstrated that esmolol, a $\beta$-adrenergic antagonist, decreases vascular retention and increases the extravascular volume. These studies suggest that adrenergic receptors play an important role on the regulation of the retention or loss of fluid after the use of NSS. However, during a hemorrhage, Moraes et al..$^{13}$ found conflicting results. These authors analyzed the effect of phenylephrine and isoproterenol during hemorrhages on vascular retention in rabbits, but no difference on volume expansion among the agonists was observed. This study showed that hemorrhage could alter the capacity that isoproterenol and phenylephrine have on intravascular fluid retention.
Dobutamine (DB) and noradrenaline (NA), the most frequent drugs used with or without infusion of crystalloids for increasing the vascular tonus and improving the cardiac function in clinical scenarios, have been found to have volume retaining properties on normovolemic sheep ${ }^{6,14}$. We hypothesized that in rabbits submitted to hemorrhage, the infusion of dobutamine, with or without an infusion of noradrenaline, impairs intravascular volume retention. We also hypothesized that the infusion of vasoactive drugs could attenuate damage to the kidneys and lungs.

\section{Methods}

After approval from the Ethics Committee for Animal Experimentation of the Botucatu Medical School, Universidade Estadual de São Paulo "Júlio de Mesquita Filho" (UNESP), 36 rabbits weighing from 2.5 to 4.5 kilograms were studied. The rabbits were provided by the Central Animal Facility of Botucatu campus of UNESP. Only normotensive animals, with free access to water and foods, were included in this study.

Anesthesia was induced with sodium thiopental (25mg/ $\mathrm{kg}$ ) in the marginal vein of the ear. After drug administration, the animals were placed on a Claude Bernard tray and the anesthesia was maintained with isoflurane given via a facial mask. Afterwards, trichotomy in the neck and abdomen, tracheotomy and intubation were performed. Isoflurane with an inspired concentration of $1.5 \%$ to $3 \%$, in $100 \% \mathrm{O}_{2}$, was used to keep the animals anesthetized.

During the whole experiment, all the animals of all groups received a hydration solution of NSS at $3 \mathrm{~mL} / \mathrm{kg} / \mathrm{h}$. Temperature was maintained using forced-air warming blankets (Bair Hugger, USA).

The animals selected for the experiment were block randomized. Each group was comprised of six animals, distributed as follows:

SHAM Group $(\mathrm{SH})$ : Animals subjected to the preparation surgical procedure without hemorrhage or volume replacement.

Control Group (CR): Animals subjected to hemorrhage of $25 \%$ of the calculated total blood volume, followed by replacement with the same volume of their own blood.

Normal Saline Group (FI): Animals subjected to hemorrhage of $25 \%$ of the calculated total blood volume and replacement with NSS at 3 times the volume of the amount of blood drawn.

Noradrenaline Group (NA): Animals subjected to hemorrhage of $25 \%$ of the calculated total blood volume and replacement with NSS at 3 times the amount of blood drawn, associated with the infusion of noradrenaline, at a dose of $1.6 \mathrm{ug} /$ 
$\mathrm{kg} / \mathrm{min}$.

Dobutamine Group (DB): Animals subjected to hemorrhage of $25 \%$ of the calculated total blood volume and replacement with NSS at 3 times the amount of blood drawn, associated with the infusion of dobutamine, at a dose of $10 \mathrm{ug} /$ $\mathrm{kg} / \mathrm{min}$.
Dobutamine + Noradrenaline Group (NADB): Animals subjected to hemorrhage of $25 \%$ of the calculated total blood volume and replacement with NSS at 3 times the amount of blood drawn, associated with the infusion of dobutamine and noradrenaline, at a dose of $10 \mathrm{ug} / \mathrm{kg} / \mathrm{min}$ and $1.6 \mathrm{ug} / \mathrm{kg} / \mathrm{min}$, respectively.

\section{Experimental protocol}

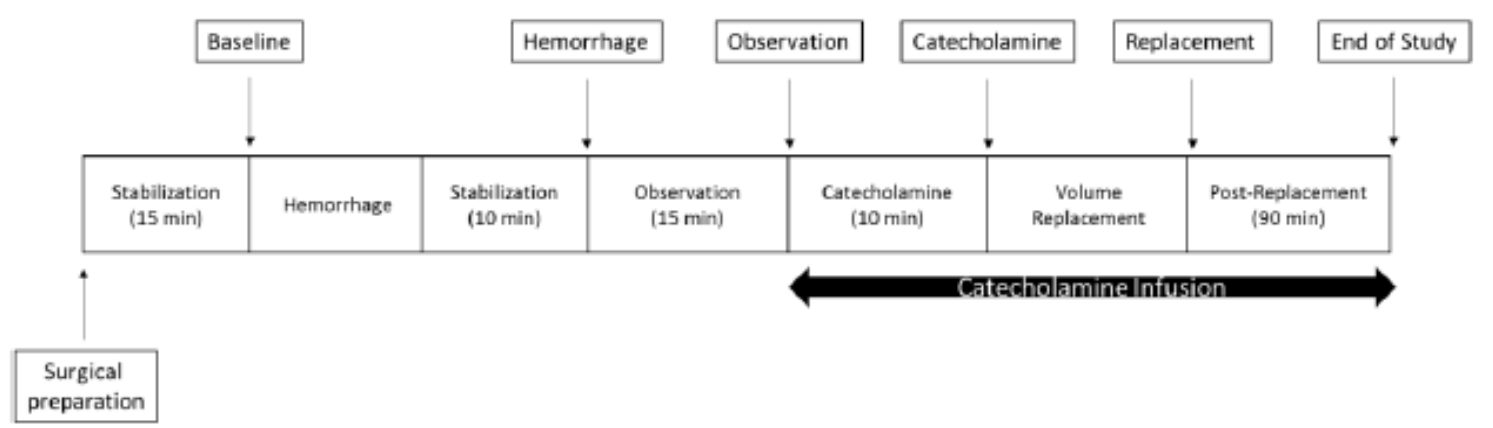

FIGURE 1 - Experimental Protocol: Baseline: 15 minutes after animal preparation; Hemorrhage: demarcated at 10 minutes after beginning of hemorrhage; Observation: 15 minutes after hemorrhage demarcation, when catecholamine infusion is begun; Catecholamine: demarcated 10 minutes after catecholamines and beginning of volume replacement; Replacement: demarcated at the end of the volemic replacement; End of study: 90 minutes after the volemic replacement.

After surgical preparation, the animals were submitted to a withdrawal of $25 \%$ of the calculated total blood volume. In order to calculate the total blood volume, we assumed $60 \mathrm{~mL}$ of blood $/ \mathrm{kg}$ of live weight ${ }^{15}$. The rate of the blood withdrawal was of $5 \mathrm{~mL} /$ minute.

After hemorrhage, the rabbits were allowed 10 minutes of stabilization, where no drug was administered, except for hydration with normal saline at a volume of $3 \mathrm{~mL} / \mathrm{Kg} / \mathrm{h}$.

After this observation period, catecholamines were started in the NA, DB, and NADB groups, diluted in normal saline for hydration maintenance.

After the end of the experiment, the animals' euthanasia was performed through a deep inhalational anesthesia associated with the administration of high doses $(100 \mathrm{mg} / \mathrm{kg})$ of intravenous sodium thiopental.

Immediately after euthanasia, samples were collected for histopathological analysis of the right inferior lobe of the lung and the left kidney. The samples were stained in hematoxylin-eosin and analyzed by a pathologist who was unaware of the group to which the animal belonged. The analysis of pulmonary and renal histology was carried out by assigning scores to histopathological lesions so that the level of alveolar or glomerular damage could be determined.

The right, middle pulmonary lobe was collected for the dry-to-wet-lung-weight ratio. The right, middle pulmonary lobes were weighed and incubated at $70^{\circ} \mathrm{C}$ for 48 hours ${ }^{16}$. At the end of this period, the material was weighed again (dry weight). The dryto-wet-lung-weight ratio was calculated to study the pulmonary water retention in these animals.

For the NGAL, the enzyme-linked immunosorbed assay (ELISA) technique was used. The plasma NGAL dosage was performed by using a NGAL ELISA kit (Triage ${ }^{\circledR}$ NGAL Device; Biosite Incorporated). The kit sensitivity was $0.02 \mathrm{ng} / \mathrm{mL}$ and the samples were analyzed in duplicate. NGAL was measured at baseline and at end of study.

The left jugular vein and right femoral artery were catheterized to measure the central venous pressure (CVP) and mean arterial pressure (MAP), respectively. Multiparametric monitors (Datex Engstrom AS3, General Electric, USA) were used to read CVP and MAP. The right jugular vein was catheterized for hydration (normal saline $3.0 \mathrm{~mL} / \mathrm{kg} / \mathrm{h}$ ) and volume replacement. The left carotid artery was also catheterized for hemorrhage and blood collection. A cystostomy was perfomed for urinary quantification in $\mathrm{mL} / \mathrm{h}$.

The hematocrit (HCT) was measured every 5 minutes throughout the experiment. Each sample was centrifuged (SPINLAB Mod: SL-MH) at a ratio of 10,000 revolutions per minute (rpm) for 5 minutes, determining the volume ratio of erythrocyte in the blood. Hematocrit was measured at each time point during the study, and the value of the hematocrit was the 
average value obtained for the two capillary tubes.

Evaluation time points:

Baseline: 15 minutes after animal preparation;

Hemorrhage: 10 minutes after beginning of hemorrhage;

Observation: 15 minutes after beginning of hemorrhage; the beginning of the catecholamine infusion;

Catecholamine: 10 minutes after beginning catecholamines; the beginning of volume replacement;

Replacement: end of the volemic replacement;

End of study: 90 minutes after the volemic replacement.

Microscopy of the kidneys was analyzed using a binocular optical microscope, examining several morphological components: the interstitium, vessels and glomeruli. The following features were assessed: edema, congestion, necrosis, inflammatory infiltrate and endothelial injury, scoring from 0 to 4 plus signs $(+)$ and focal or diffuse pattern.

Microscopy of the lungs was analyzed using a binocular optical microscope, examining several morphological components: alveolar space, alveolar wall and vessels. The following features were assessed: edema, congestion, necrosis, inflammatory infiltrate and endothelial injury, scoring from 0 to 4 plus signs $(+)$ and focal or diffuse pattern.

\section{Statistical analysis}

The statistical analysis of the results was performed using the Profile Analysis ${ }^{17}$ of the Statistical Package for the Social Sciences (SPSS) computer program, version 17.0.The F statistics were considered significant when $p<0.05$, where $p$ is the significance level associated with the calculated statistics.

The Shapiro-Wilk and Levene tests were used to test normality and homogeneity of variance respectively.

For the variables with normal distribution and homogeneity of variances, profile analysis was used; otherwise, Friedman's test was carried out for comparing the moments in each group and the Kruskal-Wallis test was used to compare the groups in each moment. For HCt, MAP and diurese variables, general linear model was used and presented the results using means and standard deviations. The comparison were made for means of groups.

For NGAL, Friedman and Kruskal-Wallis tests were used and presented the results using box-plots.

All the moments were considered for making up the hematocrit curve profile; however, for the statistical analysis, the following moments were considered: Baseline (Bas), Hemorrhage (Hem), Observation (Obs), Catecholamine (Cat), Replacement (Repl), End of Study (End).

\section{Results}

None of the animals died during the experiment.

The intravascular volume retention was not different among the groups (Figure 2). As expected, HCT dropped after hemorrhage; nevertheless, only after volume replacement were the HCT values significantly different from the CR and SHAM groups (Figure 3). In all groups subjected to hemorrhage, MAP decreased during the hemorrhagic period, returning to baseline levels after volume replacement (Figures 4).

As for the renal evaluation, diuresis was not different between the groups or the moments until volume replacement. After volume replacement, there was a progressive and significant increase in the urine output, especially in the group subjected to dobutamine (Figure 5). Nevertheless, the NGAL values were not significantly different between the analyzed groups and moments (Figure 6). The kidney histological analysis corroborates these findings with no significant damage regardless of the presence of hemorrhage or use of catecholamines. No changes were observed in any of the kidney samples from the different groups.

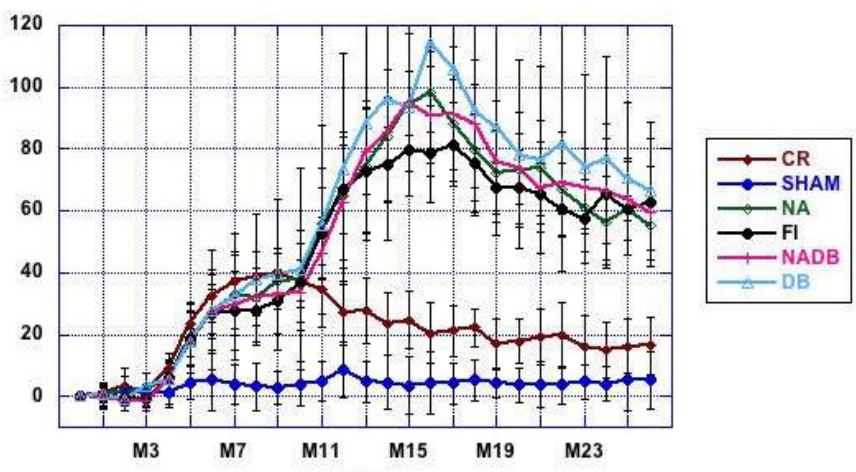

FIGURE 2 - The volemic variation ( $y$-axis) was calculated by based on body weight, average values of the hematocrit in each moment of the experiment (as delineated on the $x$-axis) and on the basal volemia based on $60 \mathrm{~mL} / \mathrm{kg}$ of body weight.

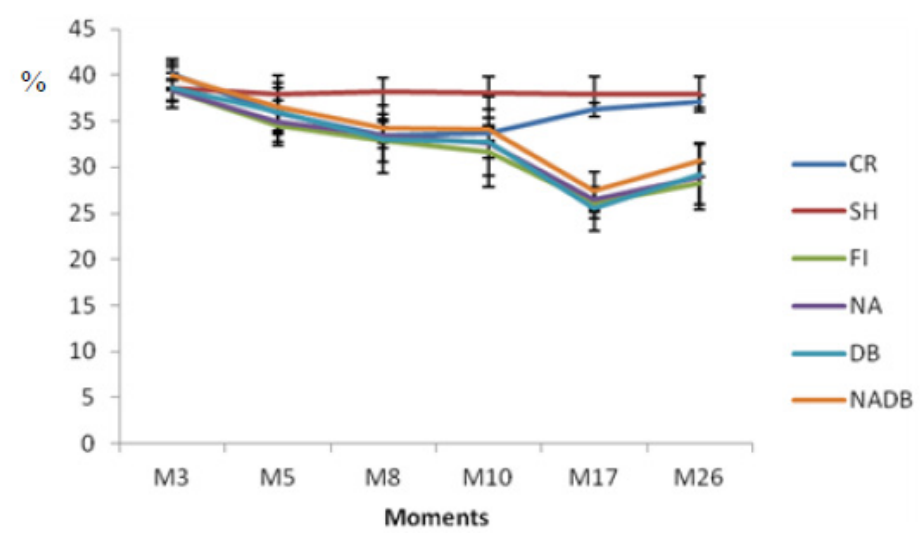

FIGURE 3 - Mean \pm standard deviation values of HCT, according to the moment and group. ( $<<0.001$ for groups, moments and interaction effects) The HCT values of the SH and CR groups differ from the others. 


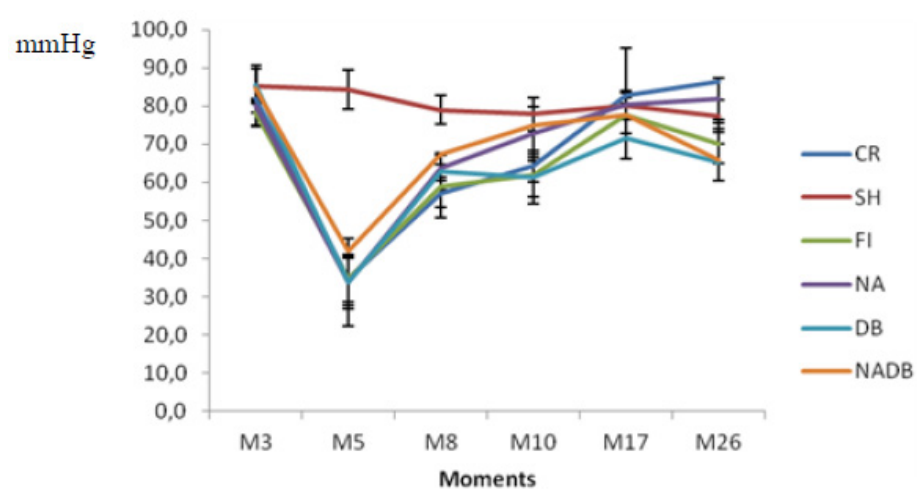

FIGURE 4 - Mean \pm standard deviation values of MAP, according to the moment and group. ( $\mathrm{p}=0.04$ for groups effect, $\mathrm{p}<0.001$ for moment effect and $\mathrm{p}=0.02$ for interaction effect).

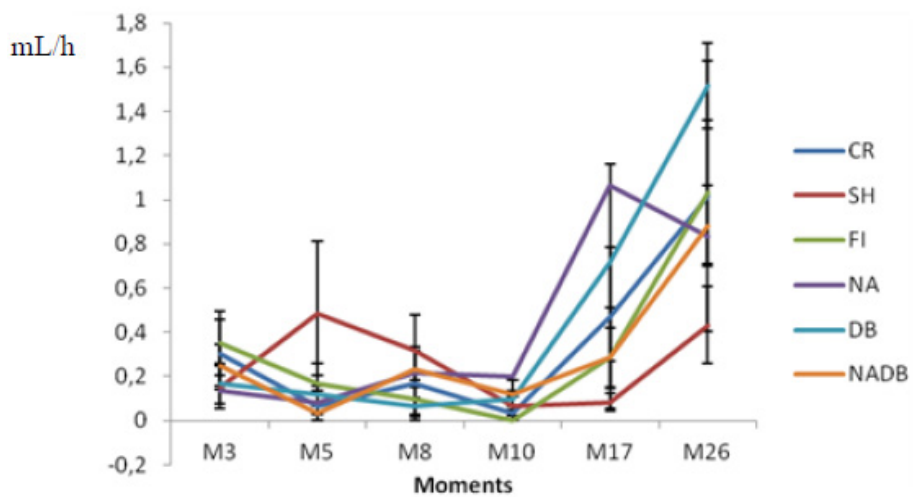

FIGURE 5 - Mean \pm standard deviation values of diuresis according to the moment and group. ( $\mathrm{p}=0.76$ for groups effect, $\mathrm{p}<0.001$ for moment and $\mathrm{p}=0.38$ for interaction effect).

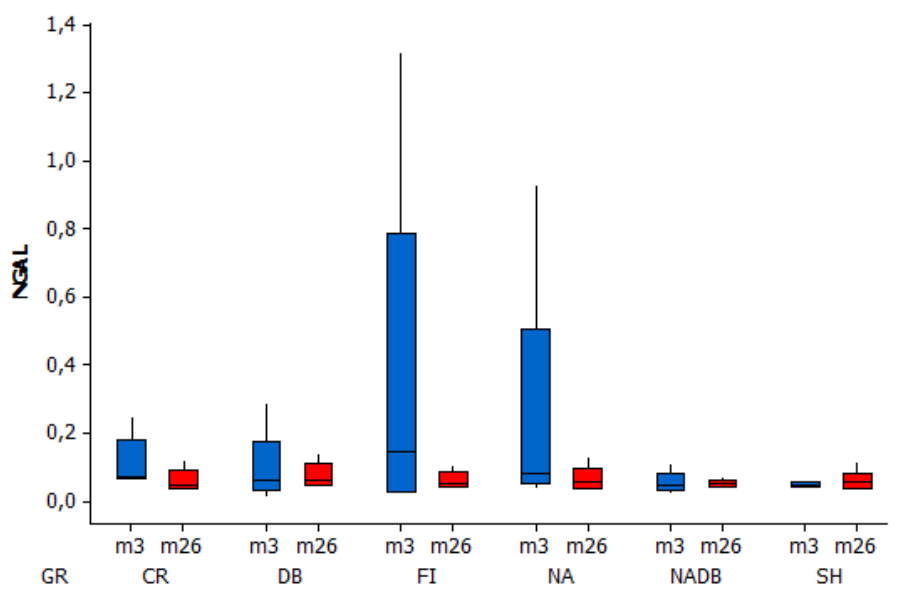

FIGURE 6 - Box plot. NGAL by moment and group ( $>>0.05)$. There was no difference among the groups in each given moment, nor among the moments in each group.
In the analysis of pulmonary tissue, diffuse alveolar congestion as well as focal hemorrhage were observed in the groups subjected to hemorrhage, regardless of the presence of catecholamines (Figure 7). The dry-to-wet-lung ratio was not different among the groups (Figure 8).

The calculation of volemic variation was made from the hematocrit dilution value. The volemic variation was calculated by based on body weight, average values of the hematocrit in each moment of the experiment and on the basal volemia based on 60 $\mathrm{mL} / \mathrm{kg}$ of body weight ${ }^{15}$.
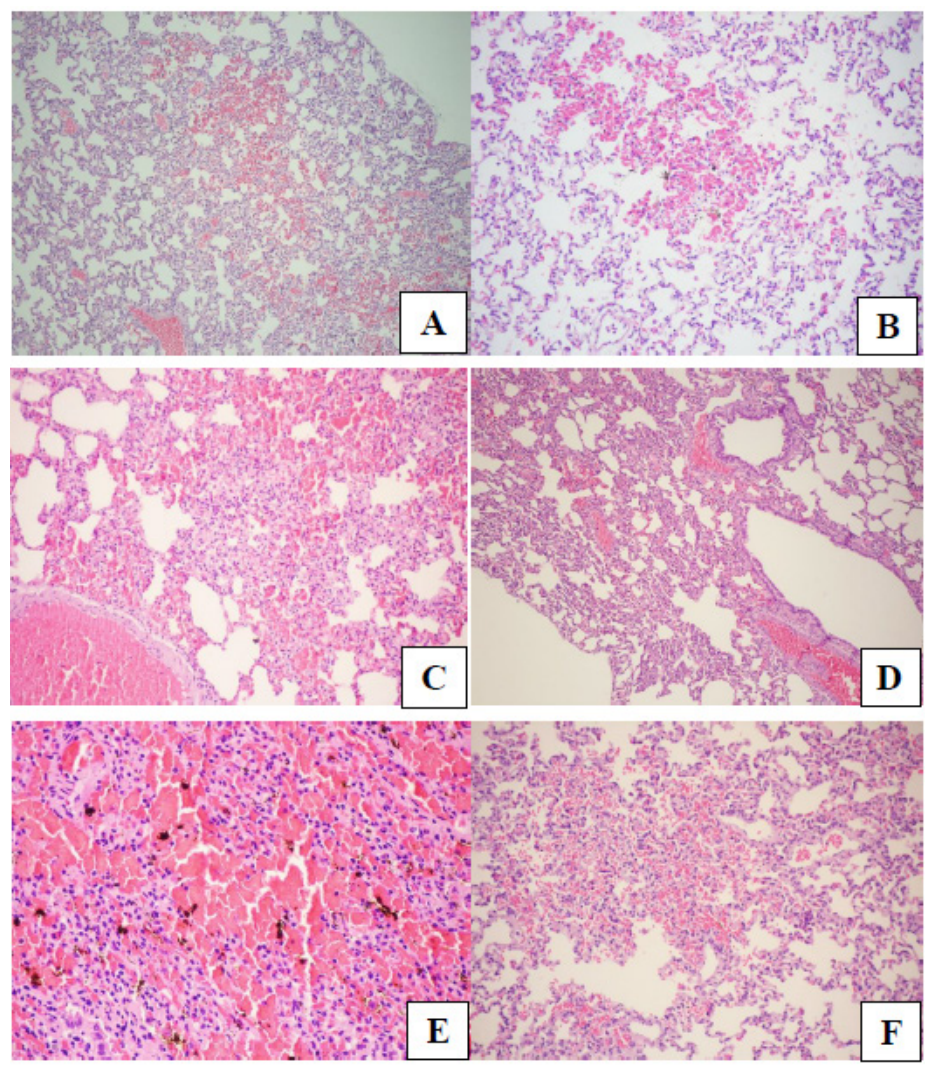

FIGURE 7 - Pulmonary microscopy (HE stain).

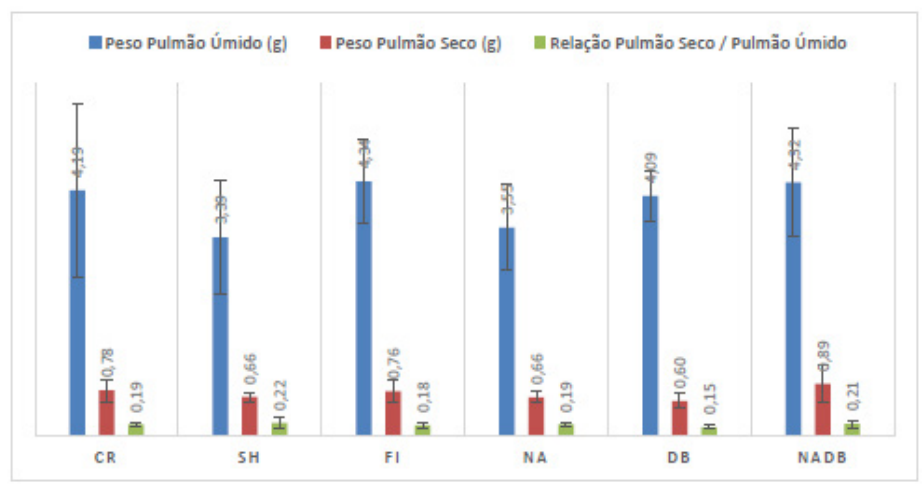

FIGURE 8 - Average and standard deviation in dry and wet lung weight, according to the moment and group. 


\section{Discussion}

The main finding of this study was that the use of noradrenaline, dobutamine or both does not cause a significant difference in the intravascular fluid retention in animals subjected to hemorrhage. Furthermore, the use of normal saline (NSS) with or without noradrenaline and dobutamine or the use of autologous blood did not cause additional damage to the kidneys and lungs.

The perioperative fluid therapy in critically ill patients aims to restore blood volume so that the tissue delivery of oxygen $\left(\mathrm{DO}_{2}\right)$ can be increased to a level that is suitable for systemic metabolic needs. In order to attain this objective, crystalloid solutions, colloid solutions or hemoderivates are given, crystalloid solutions being the first choice. The main disadvantage of these solutions is that they only remain in the intravascular space in small amounts, causing redistribution to the extracellular space. This leads to a translocation of nearly $4 / 5$ of the administered volume to the interstitium, resulting in edema and tissue hypoxia ${ }^{4}$.

On the other hand, the restoration of adequate oxygen supply to the tissues is frequently followed by the administration of inotropic or vasoactive solutions. For example, the behavior of crystalloid solutions infused into normovolemic and conscious sheep given $\alpha$-, $\beta$ - and dopamine agonist catecholamines have been studied ${ }^{11,12}$. The authors of these studies concluded that the $\beta$-agonist catecholamines and dopamine are able to cause an intravascular retention of crystalloid solutions, whereas the $\alpha$-agonist catecholamines depleted the intravascular volume at a greater intensity, mostly due to a higher diuretic effect ${ }^{11,12}$.

A similar, yet still very different study done by Vane et al. ${ }^{11}$ found that there was higher water retention after normal saline infusion associated with a $\beta$ - agonist drug. The current study, however, was carried out in animals already exposed to systemic inflammatory process because of a hemorrhagic shock, and therefore it looked at a very different reaction. The studies by Vane et al. ${ }^{11}$ and Stephens et al. ${ }^{14}$ were performed in hygid and normovolemic animals, which were not subjected to hemorrhage. Therefore, the ischemia-reperfusion injury, systemic inflammation and other unknown process might have been responsible for the alteration in intravascular volume dynamics associated with catecholamines.

Other studies have found that the tissue hypoxia arising from a $\mathrm{DO}_{2}$ reduction after hemorrhagic shock leads to the release of inflammatory mediators ${ }^{5,18,19}$, which, in turn, cause a decrease in the endothelial glycocalyx, resulting in the plaque aggregation ${ }^{20}$, leukocyte adhesion on the endothelial surface ${ }^{21}$, and an increase in the endothelial permeability causing tissue edema ${ }^{22,23}$. Furthermore, experimental studies have shown that in the ischemia-reperfusion process $^{24}$, the proteases ${ }^{25}$, the tumor necrosis factor- $\alpha(T N F-\alpha)^{26}$, the oxygen free radicals ${ }^{20}$ and the atrial natriuretic peptide (ANP) are able to degrade the endothelial glycocalyx. Our study, in agreement with what Moraes et al. ${ }^{13}$ found in their study of volemic replacement after a hemorrhagic shock and the use of pure $\alpha$ - and $\beta$ - agonists, shows that non-specific $\alpha$ - and $\beta$ - agonists also have the same behavior as pure agonists under hemorrhagic shock.

Another important finding of this study was the lack of significant histological differences and the lack of differences in dry- to-wet-lung-weight ratio when the animals were treated with NSS or with blood. This finding helps corroborate the recent studies that have demonstrated an increase in the morbid-mortality rates in patients who received blood transfusion when compared with those who did not ${ }^{27}$. Blood transfusion has been associated with higher rates of infection, sepsis, pulmonary complications and thromboembolic events ${ }^{28}$. Thus, our findings show that for lung and kidney lesions with up to this level of hemorrhage, withholding packed red cell transfusions and increasing vasoactive support may provide similar results.

The use of volemic replacement solutions with or without vasopressors or cardiostimulants in animals present several limitations. The main limitations are due to the drugs' physiological responses and dosages, and to the lower blood volume of the animals as compared to humans. Since the total blood volume of rabbits is much lower than humans, an approximation using the amount of blood per kilogram was used. Under these circumstances, withdrawing $25 \%$ of the calculated total blood of the animal might not have the same physiological response in humans. Another limitation lies with the model of hemorrhage, which was done by withdrawing blood directly from an artery. In clinical scenarios, tissue lacerations and wounds are what cause blood loss, which could pose different physiological responses, including different inflammatory profiles.

Catecholamines, especially epinephrine, can mobilize red blood cells into circulation from the spleen ${ }^{29}$. Since the rabbits were not splenectomized, this could have influenced the results of this study. However, most hemorrhagic shocks do not occur in splenectomized patients. Based on this fact, the authors opted not to perform another surgical operation; that said, the rabbits' spleens could have skewed the results of the study.

This article aims specifically the replacement with normal saline solution. We would not know the vascular retention 
behavior of other volemic replacement fluids when compared to the catecholamines used.

Another limitation is due to the use of the dry-to-wetlung- weight ratio. This method may have a low sensitivity and be unable to detect low levels of pulmonary edema in animals with intra-alvelolar hemorrhage, and alveolar and interstitial edema.

Our goal was to evaluate the effects of pharmacological agents on the volemic retention in hemorrhagic conditions. It is likely that the effects that were observed in this animal model could be different in other animal models or even in the same animal under different conditions, such as a more severe hemorrhage or sepsis. Further studies will be needed to determine whether these agents, in clinical doses, have similar effects during resuscitation.

\section{Conclusions}

There was no major intravascular volume retention after the normal saline infusion with the use of noradrenaline, dobutamine or their combination. Furthermore, the NSS used with noradrenaline, dobutamine, both, or autologous blood does not present additional damage to the kidneys or lungs.

\section{References}

1. Shoemaker WC, Peitzman AB, Bellamy R, Bellomo R, Bruttig SP, Capone A et al. Resuscitation from severe hemorrhage. Crit Care Med. 1996;24:S12-3. PMID: 8608703.

2. Alexander RH. Shock, Advanced Trauma Life Support: Program for Physicians, Instructor Manual. In: American College of Surgeons, ed. Advanced Trauma Life Support. Chicago, IL: American College of Surgeons; 1993. p.75-94.

3. Wagner BK, D'Amelio LF. Pharmacologic and clinical considerations in selecting crystalloid, colloidal, and oxygen-carrying resuscitation fluids, Part 1. Clin Pharm. 1993;12(5):335-46. PMID: 3097557.

4. Tølløfsrud S, Tønnessen T, Skraastad O, Noddeland H. Hypertonic saline and dextran in normovolemic and hypovolemic healthy volunteers increases interstitial and intravascular fluid volumes. Acta Anaesthesiol Scand. 1998;42:145-53. PMID: 9509194.

5. Tølløfsrud S, Mathru M, Kramer GC. Hypertonic-hyperoncotic solutions in open-heart surgery. Perfusion. 1998;13:289-96. PMID: 9778711.

6. Lowell JA, Schifferdecker C, Driscoll DF, Benotti PN, Bistrian BR. Postoperative fluid overload: not a benign problem. Crit Care Med. 1990;18:728-33. PMID: 2364713.

7. Demling R, Harms B, Kramer G, Gunther R. Acute versus chronic hypoproteinemia and post-traumatic pulmonary edema. Surgery. 1982;92:79-86. PMID: 7089871.

8. Holmes C. Vasoactive drugs in the intensive care unit. Curr Opin Crit Care. 2005;11:413-7. PMID: 16175026.

9. Bellomo R, Wan L, May C. Vasoactive drugs and acute kidney injury. Crit Care Med. 2008;36 S179-86. PMID: 18382191.

10. Al-Hesayen A, Parker JD. The effects of dobutamine on renal sympathetic activity in human heart failure. J Cardiovasc Pharmacol. 2008;51:434-6. PMID: 18398381.
11. Vane LA, Prough DS, Kinsky MA, Williams CA, Grady JJ, Kramer GC. Effects of different catecholamines on the dynamics of volume expansion of crystalloid infusion. Anesthesiology. 2004;101:113644. PMID: 15505449.

12. Kinsky MP, Vaid SU, Vane LA, Prough DS, Kramer GC: Effect of esmolol on fluid therapy in normovolemia and hypovolemia. Shock. 2008;30:55-63. PMID: 18323741.

13. Moraes JM, Vane MF, Rodrigues Dde F, Mostarda CT, Moraes TS, Vane LF, Ganem EM, Cavalcanti IL, Módolo NS, Vane LA. Effects of catecholamines on volemic replacement with saline solution and the impact on heart rate variability in rabbits subjected to hemorrhage. A study by spectral analysis. Acta Cir Bras. 2014;29:703-10. PMID: 25424289.

14. Stephens CT, Uwaydah N, Kramer GC, Prough DS, Salter M, Kinsky MP. Vascular and extravascular volume expansion of dobutamine and norepinephrine in normovolemic sheep. Shock. 2011;36:30311. PMID: 21654559.

15. Little RA. Changes in the blood volume of the rabbit with age. J Physiol. 1970;208:485-97. PMID: 5500739.

16. Miserocci G. Mechanisms controlling the volume of pleural fluid and extravascular lung water. Eur Respir Rev. 2009;18(114):244-52. PMID: 20956149.

17. Morrison DF. Multivariate statistical methods. New York: McGrawHill; 1967.

18. Adamson RH. Permeability of frog mesenteric capillaries after partial pronase digestion of the endothelial glycocalyx. J Physiol. 1990;428:1-13. PMID: 2231409.

19. Wilmore DW. Metabolic response to severe surgical illness: overview. World J Surg. 2000;24:705-11. PMID: 10773123.

20. Vink H, Constantinescu AA, Spaan JAE. Oxidized lipoproteins degrade the endothelial surface layer: implications for plateletendothelial cell adhesion. Circulation. 2000;101:1500-2. PMID: 10747340.

21. Constantinescu AA, Vink H, Spaan JAE. Endothelial cell glycocalyx modulates immobilization of leukocytes at the endothelial surface. Arterioscler Thromb Vasc Biol. 2003;23:1541-7. PMID: 12855481.

22. Chappell D, Jacob M, Hofmann-Kiefer K, Bruegger D, Rehm M, Conzen P, Welsch U, Becker BF. Hydrocortisone preserves the vascular barrier by protecting the endothelial glycocalyx. Anesthesiology. 2007;107:776-84. PMID: 18073553.

23. Jacob M, Bruegger D, Rehm M, Stoeckelhuber M, Welsch U, Conzen $\mathrm{P}$, Becker BF. The endothelial glycocalyx affords compatibility of Starling's principle and high cardiac interstitial albumin levels. Cardiovasc Res. 2007;73:575-86. PMID: 17196565.

24. Rehm M, Orth V, Kreimeier U, Brechtelsbauer H, Finsterer U. Changes in intravascular volume during acute normovolemic hemodilution and intraoperative retransfusion in patients with radical hysterectomy. Anesthesiology. 2000;92:57-64. PMID: 11046202

25. Adamson RH, Lenz JF, Zhang X, Adamson GN, Weinbaum S, Curry FE. Oncotic pressures opposing filtration across non-fenestrated rat microvessels. J Physiol. 2004;557:889-907. PMID: 15073281.

26. Henry CB, Duling BR. TNF-alpha increases entry of macromolecules into luminal endothelial cell glycocalyx. Am J Physiol Heart Circ Physiol. 2000;279: H2815-23. PMID: 11087236.

27. Glance LG, Dick AW, Mukamel DB, Fleming FJ, Zollo RA, Wissler R. Association between intraoperative blood transfusion and mortality and morbidity in patients undergoing noncardiac surgery. Anesthesiology. 2011;114:283-92. PMID: 21239971.

28. Bydon M, Abt NB, Macki M, Brem H, Huang J, Bydon A Preoperative anemia increases postoperative morbidity in elective cranial neurosurgery. Surg Neurol Int. 2014;5:156. PMID: 25422784 
Ramalho GL et al.

29. Hannon JP, Bossone CA, Rodkey WG. Splenic red cell sequestration and blood volume measurements in conscious pigs. Am J Physiology. 1985;248:R293-301. PMID: 3976907.

\section{Correspondence:}

Luiz Antonio Vane

Departamento de Anestesiologia

Faculdade de Medicina de Botucatu - UNESP

Caixa Postal 530

18618-970 Botucatu - São Paulo Brasil

lavane@fmb.unesp.br

Received: May 20, 2016

Review: July 18, 2016

Accepted: Aug 21, 2016

Conflict of interest: none

Financial source: none

${ }^{1}$ Research performed at Experimental Laboratory of Anesthesiology, Botucatu Medical School, Universidade Estadual Paulista (UNESP), Brazil. PhD degree thesis, Postgraduate Program in Anesthesiology, UNESP. Tutor: Luiz Antonio Vane. 